\title{
On Two Types of Surfaces of General Type with Vanishing Geometric Genus
}

\author{
C.A.M.Peters ${ }^{\star}$ (Cambridge, Mass.)
}

\section{Notation and Terminology}

If $S$ is a complex manifold we put

$\mathcal{O}_{S}:$ the structure sheaf of $S$,

$\Theta_{S}$ : the sheaf of germs of holomorphic vector fields on $S$,

$\Omega_{S}^{p}$ : the sheaf of germs of holomorphic $p$-forms on $S$,

$K_{S}$ : the canonical bundle of $S$ (i.e. the bundle of holomorphic $m$-forms, $m=\operatorname{dim}_{\mathbb{C}} S$ ) or also the canonical divisor on $S$.

If $F$ is any holomorphic vector bundle on $S$ we let $\mathscr{O}_{S}(F)$ be the corresponding sheaf of germs of holomorphic sections. For any coherent sheaf $G$ on $S$ we put

$$
\begin{aligned}
& h^{p}(G)=\operatorname{dim}_{\mathbb{C}} H^{p}(S, G), \\
& \chi(G)=\text { Euler characteristic of } G .
\end{aligned}
$$

We use the standard notations

$$
\begin{aligned}
& p_{g}(S)=h^{0}\left(K_{S}\right) \quad \text { (geometric genus), } \\
& P_{m}(S)=h^{0}\left(K_{S}^{\otimes m}\right) \quad m \geqq 2 \quad \text { ( } m \text {-th plurigenus), } \\
& q(S)=h^{0}\left(\Omega_{S}^{1}\right) \quad \text { (irregularity). }
\end{aligned}
$$

If $S \subset \mathbb{P}^{N}$ we abbreviate $\mathcal{O}_{S}\left(F \otimes H^{l}\right)=\mathcal{O}_{S}(F(l))$ for $H$ the hyperplane bundle of $\mathbb{P}^{N}$.

If $D$ is any divisor on $S$ let $|D|$ be the corresponding linear system. If $|D| \neq \phi$ we can pick a basis $d_{0}, \ldots, d_{n}$ for $h^{0}(D)$ and define the rational map $\Phi_{D}: S \rightarrow \mathbb{P}^{n}$ by assigning to the point $x$ the point $\Phi_{D}(x)$ in $\mathbb{P}^{n}$ with homogeneous coordinates $\left(d_{0}(x), \ldots, d_{n}(x)\right)$. We abbreviate $\Phi_{m K}=\Phi_{m}$ and we say that $S$ is of general type if $\Phi_{m}$ is birational onto its image for big $m$. Finally if $S$ is a surface we let $c_{1}^{2}$ and $c_{2}$ be the degrees of the Chern classex $c_{1}^{2}$, resp. $c_{2}$ and Tor $(S)$ the torsion subgroup of the Neron Severi-group.

\footnotetext{
* Supported by the Netherlands Organization for the Advancement of Pure Research (Z.W.O.).
} 


\section{§ 0. Introduction}

The classical criterion of Castelnuovo for the rationality of a complex algebraic surface $S$ states that $S$ is rational (i.e. birationally equivalent to $\mathbb{P}^{2}$ ) if and only if $P_{2}=q=0$. It is therefore natural to ask for the existence of non-rational surfaces with $p_{g}=q=0$. Perhaps the oldest example is due to Enriques and this surface still bears his name. See Kodaira, [8] for more details. Here we only remark that $c_{1}^{2}$ is zero for this surface. Campedelli seems to be the first to give examples of non-rational surfaces with $p_{g}=q=0$, but $c_{1}^{2}>0, P_{2} \neq 0$. See [6]. From the general classification theory of Kodaira [8] it follows that such a surface is of general type. Later on Godeaux [7] constructed other such surfaces. Until very recently no other surfaces of general type with $p_{g}=q=0$ were known. ${ }^{1}$ The study of the torsion groups however led $\mathrm{M}$. Reid to the construction of some new examples, see [10]. It turns out that his ideas can be applied fruitfully to compare the Campedelli surface and one of the Godeaux surfaces. Apart from giving a modern treatment of the surfaces in question this is the main purpose of this paper. We find that the Campedelli surface can be deformed into some Godeaux surface. We derive this from a more precise result concerning the deformations of the Campedelli surface (Theorem 6).

In order to motivate the computations for the Godeaux surface in question we constantly compare it with a much simpler Godeaux surface.

We give the relevant constructions in $\S 1$. The multi-canonical mappings are studied in $\S 2$. We confirm a conjecture of Bombieri, namely that $\Phi_{3}$ is birational for the Campedelli surface. (See Bombieri, [2]). In $\S 3$ we give the main result connecting the Campedelli and Godeaux surfaces. As a by-product we find the fundamental group of the Campedelli surface. In $\S 4$ we study the deformations of the various surfaces.

Acknowledgements. I want to thank Ph. Griffiths and M. Cornalba for various stimulating and helpful conversations, D. Mumford for generously showing me his correspondence with M. Reid which provided me with the fundamental ideas underlying this paper, E. Horikawa for helpful criticism of some of my earlier ideas, and finally a referee for remarks concerning the history.

\section{§1. Three Examples}

Example 1. We follow Bombieri, [1] Section 7. The group $\mathbb{Z}_{5}$ operates on $\mathbb{P}^{3}$ as follows. Let $\varepsilon=\exp (2 \pi i / 5)$, put

$$
e\left(x_{1}, x_{2}, x_{3}, x_{4}\right)=\left(x_{1}, \varepsilon x_{2}, \varepsilon^{2} x_{3}, \varepsilon^{3} x_{4}\right)
$$

This action has as fixed points precisely the coordinate vertices $(1,0,0,0),(0,1,0,0)$, $(0,0,1,0)$, and $(0,0,0,1)$. Choose any $e$-invariant smooth surface $S^{\prime}$ of degree 5 not passing through the coordinate vertices. $T$ he group $\mathbb{Z}_{5}$ then operates freely and without fixed points on $S^{\prime}$, hence the quotient $S$ is smooth. We have $p_{g}\left(S^{\prime}\right)=$ $h^{0}\left(K_{S^{\prime}}\right)=h^{0}\left(H_{S^{\prime}}\right)=4$ and $q\left(S^{\prime}\right)=0$. Because $\chi\left(\mathcal{O}_{S^{\prime}}\right)=5 \chi\left(\mathcal{O}_{S}\right)$, one has that $\chi\left(\mathcal{O}_{S}\right)=1$. Now $q(S)=0$, hence $p_{g}(S)=0$. To calculate $c_{1}^{2}(S)$, observe that $\chi\left(\mathcal{O}_{S}\right)=\frac{1}{5} \chi\left(\mathcal{O}_{S^{\prime}}\right)$,

$1 \quad$ P. Burniat "Sur les surfaces de genre $P_{12}>0$ ", Ann. Math. Pura Appl. (4) 71 (1966), claims to have constructed surfaces with $p_{\mathrm{g}}=q=0, c_{1}^{2}=2,3,4,5,6$. 
and $c_{2}(S)=\frac{1}{5} c_{2}\left(S^{\prime}\right)$ - the latter being equal to the Euler-Poincare characteristic of the surface. So Riemann-Roch gives that

$$
c_{1}^{2}(S)=\frac{1}{5} c_{1}^{2}\left(S^{\prime}\right)=\frac{1}{5}\left(H_{S^{\prime}} \cdot H_{S^{\prime}}\right)=\frac{1}{5} \operatorname{deg} S^{\prime}=1 .
$$

Remark 1. M. Reid [10] points out how the construction of the Godeauxsurfaces is related to the torsion group. Let $S$ be any surface with invariants $p_{g}=q=0, c_{1}^{2}=1$, $\operatorname{Tor}(S)=\mathbb{Z}_{5}$. Denote the elements of $\mathbb{Z}_{5}$ by $0,1,2,3$, and 4 . Reid shows that $h^{0}\left(K_{S}+i\right) \neq 0(i=1, \ldots, 4)$. Choose non-zero sections $x_{i} \in H^{0}\left(K_{S}+i\right)$ $(i=1, \ldots, 4)$, then any 5 -th degree monomial in the $x_{i}$ gives a section of $5 K_{s}$. By calculation of the respective numbers one shows that there is exactly one relation between these monomials. If $\pi: Y \rightarrow X$ is the cyclic unbranched covering of degree 5 associated to $\mathbb{Z}_{5}$ and one denotes $y_{i}=\pi^{*}\left(x_{i}\right)$ then the $y_{i}(i=1, \ldots, 4)$ form a basis for $H^{0}\left(Y, K_{Y}\right)$ and they define the birational map $\Phi_{1}: Y \rightarrow \mathbb{P}^{3}$. The image is a 5 -th degree surface $Y_{1}$ in $\mathbb{P}^{3}$. If $Y$ is non-singular one recovers a Godeaux surface. We shall use such a construction in case of the Campedelli surface.

Example 2 (cf. Godeaux, [7]). The group $\mathbb{Z}_{8}$ operates in a similar way on $\mathbb{P}^{6}$ with fixed points the coordinate vertices. We put $G=\mathbb{Z}_{8}$. Let $T$ be the complete intersection of 4 quadrics in $\mathbb{P}^{6}$ chosen in the following way:

(1) no quadric passes through the coordinate vertices

(2) the ideal generated by the 4 quadrics is $G$-invariant. As before the quotient $T$ is a smooth surface and calculation of the invariants gives:

$$
p_{g}(T)=q(T)=0, \quad c_{1}^{2}(T)=2 .
$$

We shall show how to construct such a $T$ explicitly. We choose 4 general quadrics $F_{1}, F_{2}, F_{3}, F_{4}$ such that $e\left(F_{v}\right)=\varepsilon^{2 v-1} . F_{v}(v=1, \ldots, 4)$, where $\varepsilon=\exp (2 \pi i / 8)$. We put $H=F_{1}+F_{2}+F_{3}+F_{4}$. We take $H, e(H), e^{2}(H)$, and $e^{3}(H)$ for the four quadrics. Then (2) is trivially fulfilled. We observe that $F_{1}$ is a linear combination of

$$
\begin{aligned}
& x_{1}^{2}, x_{3} x_{7}, x_{4} x_{6} \text { and } x_{5}^{2} ; \quad F_{2} \text { of } \\
& x_{2}^{2}, x_{1} x_{3}, x_{5} x_{7} \text { and } x_{6}^{2} ; \quad F_{3} \text { of } \\
& x_{3}^{2}, x_{1} x_{5}, x_{2} x_{4} \text { and } x_{7}^{2} \text {; finally } F_{4} \text { of } \\
& x_{4}^{2}, x_{1} x_{7}, x_{2} x_{6} \text { and } x_{3} x_{5} \text {. }
\end{aligned}
$$

So it is possible to let all squares appear in $H$ and hence in $e(H), e^{2}(H)$ and $e^{3}(H)$, so that (1) is fulfilled. Finally we have the equality of ideals

$$
\left(H, e(H), e^{2}(H), e^{3}(H)\right)=\left(F_{1}, F_{2}, F_{3}, F_{4}\right)
$$

and it is easy to see that $T$ is smooth in general.

Example 2 bis. A similar construction is possible for the groups $\mathbb{Z}_{4} \oplus \mathbb{Z}_{2}$ and $\mathbb{Z}_{2} \oplus \mathbb{Z}_{2} \oplus \mathbb{Z}_{2}$. See Reid, [10]. Because we want to compare the Campedelli surface $X$ with torsion $\mathbb{Z}_{2} \oplus \mathbb{Z}_{2} \oplus \mathbb{Z}_{2}$ we give the construction of $X$ explicitly. Denote the elements of $G=\mathbb{Z}_{2} \oplus \mathbb{Z}_{2} \oplus \mathbb{Z}_{2}$ by $000,100,010,001,011,101,110$ and 111. Any non-zero section of $H^{0}(K+100)$ is denoted by $x_{1}$, similarly

$$
\begin{aligned}
& x_{2} \in H^{0}(K+010), \quad x_{3} \in H^{0}(K+001), \quad y_{1} \in H^{0}(K+011), \quad y_{2} \in H^{0}(K+101), \\
& y_{3} \in H^{0}(K+110), \quad \text { and } z \in H^{0}(K+111) .
\end{aligned}
$$


All squares $x_{i}^{2}, y_{i}^{2}(i=1,2,3)$ and $z^{2}$ belong to $H^{0}(2 K)$ and there must be 4 relations among them. Let $\pi: Y \rightarrow X$ be the $G$-cyclic covering of the surface in question, $\pi^{*} x_{i}=\xi_{i}, \pi^{*} y_{i}=\eta_{i}(i=1,2,3)$ and $\pi^{*} z=\zeta$. One finds that $H^{0}\left(K_{Y}\right)$ has the $\xi_{i}, \eta_{i}$, and $\zeta$ as a basis and the map $\Phi_{1}$ gives a birational map of $Y$ onto a complete intersection $Y_{1}$ of 4 quadrics in $\mathbb{P}^{6}$. This map is biholomorphic provided $\left|K_{Y}\right|$ has no base points and $Y_{1}$ is smooth. The group $G$ acts on $\mathbb{P}^{6}$ as follows:

$$
\begin{aligned}
& (100)\left(\xi_{1}, \xi_{2}, \xi_{3}, \eta_{1}, \eta_{2}, \eta_{3}, \zeta\right)=\left(-\xi_{1}, \xi_{2}, \xi_{3}, \eta_{1},-\eta_{2},-\eta_{3},-\zeta\right), \\
& (010)\left(\xi_{1}, \xi_{2}, \xi_{3}, \eta_{1}, \eta_{2}, \eta_{3}, \zeta\right)=\left(\xi_{1},-\xi_{2}, \xi_{3},-\eta_{1}, \eta_{2},-\eta_{3},-\zeta,\right. \\
& (001)\left(\xi_{1}, \xi_{2}, \xi_{3}, \eta_{1}, \eta_{2}, \eta_{3}, \zeta\right)=\left(\xi_{1}, \xi_{2},-\xi_{3},-\eta_{1},-\eta_{2}, \eta_{3},-\zeta\right) .
\end{aligned}
$$

We see that the fixed set of $G$ is 3-dimensional, hence $X$ can be chosen disjoint from this set and smooth by a construction similar to the one in Example 2.

Our third example will be a double covering of the projective plane branched along a very specific 10-th degree curve.

First we need some preliminaries on blown-up planes. Let $P=\mathbb{P}^{2}$ and blow up $P$ at the points $p_{1}, \ldots, p_{n}$. Let $P_{1}$ be the resulting surface and let $\pi_{1}: P_{1} \rightarrow P$ be the blowing-down map. We put $E_{i}=\left(\pi_{1}\right)^{-1}\left(p_{i}\right)(i=1, \ldots, n)$ and we blow up $P_{1}$ at the point $l_{i} \in E_{i}(i=1, \ldots, n)$. We obtain $P_{2}$ and a map $\pi_{2}: P_{2} \rightarrow P_{1}$. Put $F_{i}=\left(\pi_{2}\right)^{-1}\left(l_{i}\right)$, $i=1, \ldots, n$ and denote the proper transform on $P_{2}$ of the curves $E_{i}$ by the same characters. Finally put $\pi=\pi_{1} \circ \pi_{2}$. Note the following intersection numbers on $P_{2}$.

$$
\begin{aligned}
& \left(E_{i}, E_{i}\right)=-2 \\
& \left(F_{i}, F_{i}\right)=-1 \\
& \left(F_{i}, E_{i}\right)=\left(\pi^{*} H, \pi^{*} H\right)=1 \\
& \left(E_{i}, \pi^{*} H\right)=\left(F_{i}, \pi^{*} H\right)=0 .
\end{aligned}
$$

From now on we use $\pi^{*} H$ and $H$ interchangeably. We shall frequently use the following easily verified formula for the canonical divisor $K_{2}$ on $P_{2}$ :

$$
K_{2} \sim(-3 H)+\sum_{i=1}^{n}\left(E_{i}+2 F_{i}\right)
$$

Next, assume that we are given a curve $C^{\prime} \subset P$ of degree $2 m$ with certain singularities $p_{1}, \ldots, p_{n}$. Suppose that it is possible to blow up $P$ at $p_{1}, \ldots, p_{n}$ and then $P_{1}$ at $l_{1}, \ldots, l_{n}$ etc., obtaining a surface $P_{k}$ such that

(1) the strict transform $\tilde{C} \subset P_{k}$ of $C^{\prime}$ is smooth,

(2) the divisor $\tilde{C}$ is linearly equivalent to $2 F-\sum_{i=1}^{s} G_{i}$, where $G_{i}$ are certain smooth curves which are all disjoint from $\tilde{C}$ and from each other.

Then we can construct a double covering $Q$ of $P_{k}$ having $C=\tilde{C}+\sum_{i=1}^{s} G_{i}$ as branch locus. This we see as follows. Let $\left\{U_{\alpha}\right\}$ be a coordinate covering of $P_{k}$ such that $C$ is given by the local equations $\left\{c_{\alpha}=0\right\}$. Let $\left\{f_{\alpha \beta}\right\}$ be a system of transition functions for the bundle $[F]$ corresponding to $F$, then, because of (2) $c_{\alpha}=f_{\alpha \beta}^{2} c_{\beta}$ on $U_{\alpha} \cap U_{\beta}$. Hence we find a submanifold $Q \subset[F]$ by the equations $\omega_{\alpha}^{2}=c_{\alpha}$, where $\omega_{\alpha}$ is a fibre coordinate of $[F] \mid U_{\alpha}$. Because of (1) and (2), $Q$ is smooth and is a double cover of $P_{k}$, branched along $C$. 
We apply this remark in case our singularities are triple points of type $(3,3)$, i.e. the three branches have a common tangent, which separates into three different lines after blowing up once. Assume that $p_{1}, \ldots, p_{n}$ are such singularities. Blowing up $P$ at these points we let $l_{i} \in E_{i}$ correspond to the common tangent of the branches of $C$ at $p_{i}$ and blowing up $P_{1}$ at these points we obtain $P_{2}$. One easily derives the following linear equivalence on $P_{2}$, connecting the total transform and strict transform of $C^{\prime}$ :

$$
\pi^{*} C^{\prime} \sim \tilde{C}+\sum_{i=1}^{n}\left(3 E_{i}+6 F_{i}\right)
$$

Using (1) one sees that $\left(\tilde{C}, E_{i}\right)=0(i=1, \ldots, n)$, hence $C$ does not meet $E_{i}$ and we may form the non-singular divisor $C=\tilde{C}+\sum_{i=1}^{n} E_{i}$. Let us define:

$$
F=m H-\sum_{i=1}^{n}\left(E_{i}+3 F_{i}\right)
$$

One sees that $C \sim 2 F$ and we may form the double covering $Y^{\prime}$ of $P_{2}$ branched along $C$. Let $\rho: Y^{\prime} \rightarrow P_{2}$ be the branching map. The curves $\rho^{-1}\left(E_{i}\right)=A_{i}$ are rational and moreover $\left(A_{i}, A_{i}\right)=\frac{1}{4}\left(\rho^{*} E_{i}, \rho^{*} E_{i}\right)=\frac{1}{4} \cdot 2 \cdot-2=-1$. Hence the $A_{i}$ are exceptional and may be blown down. We let $Y$ be the blown down surface and $\sigma: Y^{\prime} \rightarrow Y$ the blowing down map. Now we are ready to give our third example, due to Campedelli, [6].

Example 3. One constructs a 10-th degree curve in $P$ with six singularities of type $(3,3)$ as follows. Let $\mathbb{C}^{2} \subset P$ obtained from $P$ by deleting the line at infinity. Take in $\mathbb{C}^{2}$ two concentric circles $C_{1}$ and $C_{2}$ together with an ellipse $C_{3}$ touching them both. Together with the two circle points at infinity $C_{1} \cup C_{2} \cup C_{3}$ has six points of type $(2,2)$. It is easy to construct a 4 -th degree curve $C_{4}$ touching this curve precisely at those six points. We let $C^{\prime}=C_{1} \cup C_{2} \cup C_{3} \cup C_{4}$. For later use we remark that the six points do not lie on a conic. The surface $Y^{\prime}$ constructed by the above procedure has precisely 6 exceptional curves and we let $Y$ be as before. The surface $Y$ is the Campedelli surface.

In order to explain why $p_{g}=q=0, c_{1}^{2}=2$, one has to compare this example with the case where the curve has no singularities. We call this the corresponding smooth case. We shall prove below that in the smooth case corresponding to the Campedelli surface, one has $p_{\mathrm{g}}=6, q=0$ and $c_{1}^{2}=8$. We shall see that throwing in a triple point of type $(3,3)$ has the effect of lowering $c_{1}^{2}$ by 1 . If the triple points are in general position, each time we insert a triple point $p_{g}$ lowers 1 and $q$ stays invariant. In our case "general position" exactly means that the six points $p_{1}, \ldots, p_{6}$ do not lie on a conic. Hence $p_{g}=0, q=0, c_{1}^{2}=2$. Now we give the details.

We need two formulas which hold in case of a double covering $\rho: Z \rightarrow X$ branched along a non-singular curve $C \subset X$ with $C \sim 2 F$ :

$K_{Z} \sim \rho^{*}\left(K_{X}+F\right)$,

$H^{p}\left(Z, \rho^{*}(G)\right) \cong H^{p}(X, G) \oplus H^{p}\left(X, G \otimes F^{-1}\right) \quad$ for any vector bundle $G$ on $X$ and any integer $p$. 
The first formula is easily verified using the construction of $Z$ as given below formula (2). Formula (5) can be proved as follows: From the Leray spectral sequence it follows that $H^{p}\left(Z, \rho^{*} G\right) \simeq H^{p}\left(X, p_{*} p^{*} G\right)$ and

$$
\rho_{*} p^{*} G \cong G \otimes \rho_{*} \rho^{*} 0_{X} \cong G \otimes\left(o_{X} \ominus \mathcal{O}_{x}(-F)\right)
$$

The last equality comes from the splitting of $\rho_{*} \rho^{*} o_{X}=\rho_{*} \hat{o}_{Z}$ in pieces invariant and anti-invariant under sheet-interchange.

From (2), (3) and (4) one sees $(m=5, n=6)$ :

$$
K_{X^{\prime}} \sim p^{*}\left(2 H-\sum_{i=1}^{6} F_{i}\right)
$$

as a consequence one sees that $c_{1}^{2}\left(Y^{\prime}\right)=\left(K_{Y^{\prime}}, K_{Y^{\prime}}\right)=-4$, hence $c_{1}^{2}(Y)=-4+6=2$.

Now use (5) and (6) to compute

$$
p_{\mathrm{g}}(Y)=p_{\mathrm{g}}\left(\mathrm{Y}^{\prime}\right)=h^{0}\left(\rho^{*}\left(2 H-\sum_{i=1}^{6} F_{i}\right)\right) \text {. }
$$

We find that it is equal to $h^{0}\left(P_{2}, 2 H-\sum_{i=1}^{6} F_{i}\right)$, i.e. the number of quadrics through $p_{1}, \ldots, p_{6}$, hence equals zero. Hence $p_{g}(Y)=0$.

Finally one must compute

$$
q(Y)=q\left(Y^{\prime}\right)=h^{1}\left(\mathcal{O}_{Y^{\prime}}\right)=h^{1}\left(P_{2}, \mathscr{O}_{P_{2}}\right)+h^{\mathrm{t}}\left(P_{2}, \mathscr{O}_{P_{2}}(-F)\right)=h^{1}\left(\mathcal{O}_{P_{2}}(-F)\right) .
$$

We now use

Lemma 1. $h^{\mathrm{t}}\left(O_{F_{2}}(-F)\right)=0$.

Proof: By Serre-duality $h^{1}\left(O_{P_{2}}(-F)\right)=h^{1}\left(O_{P_{2}}\left(F+K_{2}\right)\right)=h^{1}\left(2 H-\sum_{i=1}^{6} F_{i}\right)$ by $(2)$ and (3).

The exact sequence on $P_{2}$

$$
0 \rightarrow 2 H-\sum_{i=1}^{6} F_{i} \rightarrow 2 H \rightarrow \underset{i=1}{6} \mathcal{O}_{F_{i}} \rightarrow 0
$$

gives in cohomology:

$$
0 \rightarrow H^{0}\left(2 H-\sum_{i=1}^{6} F_{i}\right) \rightarrow H^{0}(2 H) \rightarrow \mathbb{C}^{6} \rightarrow H^{1}\left(2 H-\sum_{i=1}^{6} F_{i}\right) \rightarrow 0
$$

Now $h^{0}\left(2 H-\sum_{i=1}^{6} F_{i}\right)$ is the number of conics through the six points $p_{1}, \ldots, p_{6}$, hence equals zero. Note $h^{0}(2 H)=6$, so by the above sequence $h^{2}\left(2 H-\sum_{i=1}^{6} F_{i}\right)=0$,

Corollary. $q(Y)=0$.

So we have proved the following proposition.

Proposition 2. For the Campedelli-surface $Y$ (Example 3) we have $p_{g}(Y)=$ $q(Y)=0, c_{1}^{2}(Y)=2$. 
Remark 2. This does not imply that $Y$ is of general type. However, in $\S 2$ we prove that $P_{2}(Y)=3$, and then Kodaira's classification theory [8] implies that $Y$ is of general type.

Remark 3. If there exists a 10-th degree curve with six points $\left(p_{1}, \ldots, p_{6}\right)$ of type $(3,3)$ lying on a conic the same calculation shows that for the resulting double cover (after blowing down exceptional curves), we get $p_{\mathrm{g}}=q=1, c_{1}^{2}=2$. It is not clear whether such a curve exists.

Remark 4. One can prove that throwing in double points or triple points which resolve into double points or simple points after one blowing up do not change the invariants $p_{g}, q$, and $c_{1}^{2}$.

\section{$\S$ 2. The Multicanonical Mappings}

We recall the results in Bombieri [1] for the case of the Godeaux surface of Example 1. The calculations will be entirely similar for the second example and will be given only for the surface with torsion group $G=\mathbb{Z}_{2} \oplus \mathbb{Z}_{2} \oplus \mathbb{Z}_{2}$ of Example 2 bis.

Theorem 1. For the Godeaux surface $S$ of Example 1 the following holds:

$12 K_{s} \mid$ has 4 base points,

$\left|3 K_{S}\right|$ has 2 base points and $\Phi_{3}$ is not holomorphic at those points.

$\Phi_{4}$ is not biholomorphic at the 4 base points of $\left|2 K_{S}\right|$.

Proof. From Remark 1 one sees that the sections of $m K_{S}$ are given by monomials $x_{1}^{i_{1}} x_{2}^{i_{2}} x_{3}^{i_{3}} x_{4}^{i_{4}}$ with $i_{1}+2 i_{2}+3 i_{3}+4 i_{4} \equiv 0 \bmod 5$. So a basis for $\left|m K_{\mathrm{S}}\right|$ is represented by:

$\left(x_{1} x_{4}, x_{2} x_{3}\right)$ for $m=2$,

$\left(x_{1} x_{2}^{2}, x_{1}^{2} x_{3}, x_{2} x_{4}^{2}, x_{3}^{2} x_{4}\right)$ for $m=3$,

$\left(x_{1} x_{3}^{3}, x_{1}^{2} x_{4}^{2}, x_{1}^{3} x_{2}, x_{1} x_{2} x_{3} x_{4}, x_{2}^{2} x_{3}^{2}, x_{2}^{3} x_{4}, x_{3} x_{4}^{3}\right) \quad$ for $m=4$.

Using this the assertions of the theorem can easily be verified. We refer to Bombieri, [1] for details.

Theorem 2. For the Godeaux surface $X$ of Example 2 bis the following holds: $\Phi_{2}$ is a holomorphic 8:1 map onto $\mathbb{P}_{2}$ branched along 7 lines in general position. $\Phi_{3}$ is holomorphic and birational.

Proof. From the way $X$ is constructed we see that $H^{0}\left(X, m K_{X}\right)$ is generated by monomials

$$
x_{1}^{n_{1}} x_{2}^{n_{2}} x_{3}^{n_{3}} y_{1}^{m_{1}} y_{2}^{m_{2}} y_{3}^{m_{3}} z^{r} \quad\left(n_{1}+n_{2}+n_{3}+m_{1}+m_{2}+m_{3}+r=m\right)
$$

with

$$
\begin{aligned}
& n_{1}+n_{2}+n_{3}+r \equiv 0(2), \\
& n_{1}+m_{1}+n_{2}+m_{2} \equiv 0(2), \\
& n_{1}+m_{1}+n_{3}+m_{3} \equiv 0(2) .
\end{aligned}
$$


We find the following explicit generators for low $m$ :

$$
\begin{aligned}
& x_{1}^{2}, x_{2}^{2}, x_{3}^{2}, y_{1}^{2}, y_{2}^{2}, y_{3}^{2}, z^{2} \quad \text { for } m=2, \\
& x_{1} x_{2} y_{3}, x_{1} x_{3} y_{2}, x_{2} x_{3} y_{1}, x_{1} y_{1} z, x_{2} y_{2} z, x_{3} y_{3} z, y_{1} y_{2} y_{3} \quad \text { for } m=3 .
\end{aligned}
$$

Because 4 relations between the 7 squares $\Phi_{2}$ maps $X$ holomorphically onto the intersection $\mathbb{P}$ of 4 hyperplanes in $\mathbb{P}^{6}$ branched along the intersection of the 7 coordinate hyperplanes with $\mathbb{P}$. Because $P_{2}(X)=7$ there are no relations among the given generators for $H^{0}\left(X, 3 K_{X}\right)$. One easily checks that $\Phi_{3}$ is holomorphic and is everywhere $1: 1$, hence $\Phi_{3}$ is a holomorphic birational map. Q.E.D.

Remark. If $\Phi_{3}(X)$ is non-singular it follows from the above proof that $\Phi_{3}$ is biholomorphic. We have not checked this since the calculation it involves seems discouragingly large.

Theorem 3. For the Campedelli surface $Y$ we have

(i) $\Phi_{2}$ is an 8:1-holomorphic map onto $\mathbb{P}^{2}$ of which the set-theoretic branch locus consists of 7 lines. The position of these lines is as follows: three of the lines have their intersection points on three other lines and the 7-th line is arbitrary.

(ii) $\Phi_{3}$ is a birational holomorphic map. On $Y$ the 3 curves coming from the original quadrics forming part of the branch locus of $\rho: Y^{\prime} \rightarrow \mathbb{P}_{2}$ are rational curves with selfintersection -2 . They are the only such curves on $Y$ and every $\Phi_{m}(m \geqq 2)$ blows down these 3 curves into 3 normal rational double points.

Proof. (i) We assume that projective coordinates $(x, y, z)$ are chosen in such a way that

$$
\begin{aligned}
& C_{1} \equiv\left(x^{2}+y^{2}-r^{2} z^{2}=0\right), \\
& C_{2} \equiv\left(x^{2}+y^{2}-R^{2} z^{2}=0\right), \\
& C_{3} \equiv\left(R^{2} x^{2}+r^{2} y^{2}-R^{2} r^{2} z^{2}=0\right) .
\end{aligned}
$$

Hence the six points of type $(3,3)$ on $C^{\prime}$ are the points $( \pm r, 0,1),(0, \pm R, 1)$ and $(1, \pm i, 0)$. A basis for the 4-th degree curves touching the quadrics at these 6 points is given by

$$
x^{2} C_{1}, y^{2} C_{2} \text { and } z^{2} C_{3} \text {. }
$$

Let $\tau: P \rightarrow P$ be the corresponding rational map, i.e.

$$
\tau(x, y, z)=\left(x^{2} C_{1}, y^{2} C_{2}, y^{3} C_{3}\right)
$$

The map $\tau$ becomes well defined as a holomorphic map on $P_{2}$. In the following we thus let $\bar{\tau}: P_{2} \rightarrow P$ be the holomorphic map corresponding to $\tau$. By an easy computation one shows

(1) The map $\bar{\tau}$ is generically $4: 1$.

(2) The set-theoretic banch locus of $\bar{\tau}$ consists of the three cordinate axes $u=0, v=0, w=0$ plus the lines $l_{1}, l_{2}, l_{3}$, see Fig. 1 . Here $(u, v, w)$ are projective coordinates of $P$, the target of $\bar{\tau}$.

A more accurate analysis shows that the proper transforms of $C_{1}, C_{2}$, and $C_{3}$ on $P_{2}$ map onto the points $p_{1}, p_{2}$ resp. $p_{3}$; the six curves $F_{i}$ map in pairs onto the lines $l_{j}$ and finally the curves $E_{i}$ map onto the points $q_{j}$. 


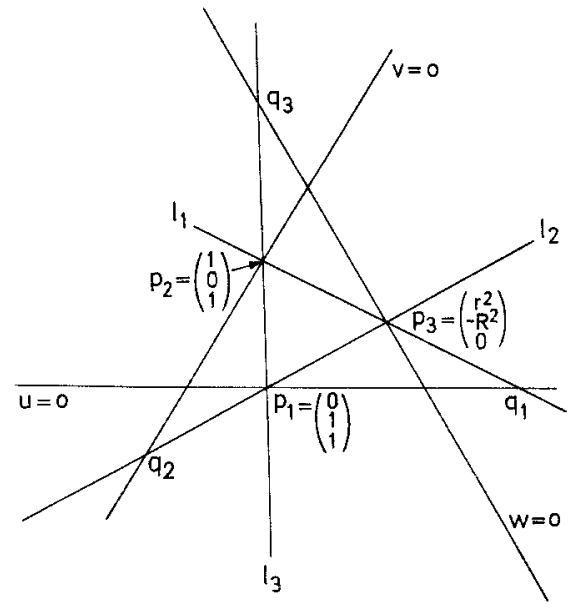

Fig. 1

In the rest of the proof we delete $\rho^{*}$ in front of divisors on $Y^{\prime}$ coming from $P_{2}$.

To see the relation with $\Phi_{2}$ we note that $2 K_{Y^{\prime}} \sim 4 H-\sum_{i=1}^{6} 2 F_{i}$ and the sections of the latter correspond in a $1-1$ way with the quartics touching the quadrics $C_{1}, C_{2}, C_{3}$ at the points $p_{1}, \ldots, p_{6}$. First of all this shows that $P_{2}=3$ and secondly one derives a commutative diagram

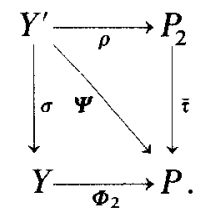

Because $\Psi\left(A_{i}\right)$ is a point we see that $\Phi_{2}$ is indeed holomorphic.

To verify the statement about the branch locus of $\Phi_{2}$ we remark that the branch locus of $\Psi$ consists of the branch locus of $\bar{\tau}$, shown in Fig. 1, and the image under $\bar{\tau}$ of the branch locus of $\rho$, hence consists of the points $p_{1}, p_{2}$ and $p_{3}$ and a general line in $P$.

(ii) To prove that $\Phi_{3}$ is birational we must prove that $\Phi_{3}$ separates points on a Zariski-dense set of $Y$. We can equally well prove that the three-canonical map of $Y^{\prime}$ has this property. Now $3 K_{Y^{\prime}} \sim 6 H-\sum_{i=1}^{6} 3 F_{i}$ and the sections of the latter are linear combinations of the ones invariant under sheet interchange and the ones anti-invariant under this operation. Recall the discussion above (5).

The invariant sections correspond to 6-tics having contact of order three with the conics $C_{1}, C_{2}$ and $C_{3}$ at the points $p_{1}, \ldots, p_{6}$. The anti-invariant sections correspond to sections of $3 K_{Y^{\prime}}-F=H+\sum_{i=1}^{6} F_{i}$, and we have $h^{0}\left(H+\sum_{i=1}^{6} F_{i}\right)=$ $h^{0}(H)=3$. We see that the invariant sections separate the fibres of the map $\rho$ 
away from the branch locus and because there are antiinvariant sections they separate the 2 points in the fibre. To see that $\Phi_{3}$ is holomorphic, notice that $|3 K|$ does not have base points, because the antiinvariant sections of $3 K_{Y^{\prime}}$ are sections of $H+\sum_{i=1}^{6} F_{i}$, hence do not have base points on $Y^{\prime}$.

Let $\tilde{C}_{i}$ be the proper transform on $P_{2}$ of the curves $C_{i}(i=1,2,3)$ and set $D_{i}=$ $\rho^{-1}\left(\tilde{C}_{i}\right)$. Then one has on $Y^{\prime}$ that

$$
\left(D_{i}, D_{i}\right)=\frac{1}{2}\left(\tilde{C}_{i}, \tilde{C}_{i}\right)=\frac{1}{2}\left(\left(C_{i}, C_{i}\right)-8\right)=-2 .
$$

Hence $D_{i}(i=1,2,3)$ is a rational curve with self-intersection -2 and the same holds for $\sigma\left(D_{i}\right), i=1,2,3$. It is easy to see that on $Y$ there are no other such curves.

In fact, any such curve $D$ must be blown down to a point under the holomorphic map $\Phi_{2}((D \cdot 2 K)=0)$ and the discussion under (i) shows that only the $D_{i}(i=1,2,3)$ are blown down to points, hence $D$ must be one of them. The other assertions now follow from Mumford [9].

\section{§ 3. The Torsion Group and a Godeaux-Type Construction for the Campedelli Surface}

The object of this section is to analyse the torsion group of the Campedelli surface and from this to obtain another construction for it.

Lemma 2. The torsion group of $Y$ contains $G=\mathbb{Z}_{2} \oplus \mathbb{Z}_{2} \oplus \mathbb{Z}_{2}$ as a subgroup.

Proof. We number the curves $E_{i}$ and $F_{i}$ in such a way that those arising from blowing up the two points not lying on $C_{i}(i=1,2,3)$ we call $E_{2 i}, E_{2 i-1}$, resp. $F_{2 i}$ and $F_{2 i-1}$. We put $D_{i}=\rho^{-1}\left(C_{i}\right),(i=1,2,3), A_{j}=\rho^{-1}\left(E_{j}\right)$ and $B_{j}=\rho^{-1}\left(F_{j}\right)(j=1, \ldots, 6)$. On $P_{2}$ we have the linear equivalences:

$$
\tilde{C}_{i} \sim 2 H-\sum_{j \neq i}\left(E_{2 j}+E_{2 j-1}+2 F_{2 j}+2 F_{2 j-1}\right) \quad i=1,2,3 .
$$

Notice that on $Y^{\prime}$ one has

$$
\rho^{*} \tilde{C}_{i} \sim 2 D_{i}, \rho^{*} E_{j} \sim 2 A_{j}, \text { and } \rho^{*} F_{j} \sim B_{j}
$$

we find:

$$
2 D_{i} \sim 2\left\{H-\sum_{j \neq i}\left(A_{2 j}+A_{2 j-1}+B_{2 j}+B_{2 j-1}\right)\right\} \quad i=1,2,3 .
$$

Now suppose that

$$
D_{i} \sim H-\sum_{j \neq i}\left(A_{2 j}+A_{2 j-1}+B_{2 j}+B_{2 j-1}\right) \quad i=1,2,3 .
$$

Then $h^{0}\left(Y^{\prime}, D_{i}\right) \neq 0$ implies that

$$
h^{0}\left(H-\sum_{j \neq i}\left(A_{2 j}+A_{2 j-1}+B_{2 j}+B_{2 j-1}\right)\right) \neq 0 .
$$


But the last number equals

$$
\begin{aligned}
h^{0}\left(H-\sum_{j \neq i} B_{2 j}+B_{2 j-1}\right) & =h^{0}\left(Y^{\prime}, \rho^{*}\left(H-\sum_{j \neq i}\left(F_{2 j}+F_{2 j-1}\right)\right)\right) \\
& =h^{0}\left(P_{2}, H-\left(\sum_{j \neq i} F_{2 j}+F_{2 j-1}\right)\right)
\end{aligned}
$$

by formula (5). But this is the number of lines through the four points $p_{j}$ lying on $C_{i}$, hence equals zero. This contradiction shows that

$$
D_{i}-H+\sum_{j \neq i}\left(A_{2 j}+A_{2 j-1}+B_{2 j}+B_{2 j-1}\right)=g_{i} \quad i=1,2,3
$$

are non-zero $\mathbb{Z}_{2}$-torsion elements. Obviously the $g_{i}$ are numerically independent, hence the $g_{i}$ generate a $\mathbb{Z}_{2} \oplus \mathbb{Z}_{2} \oplus \mathbb{Z}_{2}$ torsion subgroup of Tor $\left(Y^{\prime}\right)$, hence of Tor $(Y)$.

Lemma 3. $h^{0}\left(K+g_{i}\right)=1 \quad(i=1,2,3), h^{0}\left(K+g_{i}+g_{j}\right)=1 \quad(i \neq j, i, j=1,2,3)$ and $h^{0}\left(K+g_{1}+g_{2}+g_{3}\right)=1$.

Proof. On $Y^{\prime}, K+g_{i}=H+D_{i}+\sum_{j \neq i}\left(A_{2 j}+A_{2 j-1}\right)-\left(B_{2 i}+B_{2 i-1}\right)$ as we see from (6) and (8). Because all $D_{i}$ and $A_{j}$ have negative self-intersection:

$$
\begin{aligned}
h^{0}(H & \left.+D_{i}+\sum_{j \neq i}\left(A_{2 j}+A_{2 j-1}\right)-B_{2 i}-B_{2 i-1}\right) \\
& =h^{0}\left(H-\left(B_{2 i}+B_{2 i-1}\right)\right)
\end{aligned}
$$

$=$ the number of lines through the two points $p_{2 i}$ and $p_{2 i-1}$, hence equals 1 .

The other equalities can be checked similarly. Q.E.D.

We enumerate the elements in $G$ and in $H^{0}(Y, K+g)$ for $g \neq 0, g \in G$ as in example 2 bis. We let $\pi: Z \rightarrow Y$ be the cyclic unbranched covering of $Y$ corresponding to $G$ and we enumerate the sections of $H^{0} Z, K_{Z}$ ) corresponding to sections of $H^{0}(Y, K+g)$ for $g \neq 0, g \in G$ again in the same way as in example 2 bis.

Lemma 4. $p_{g}(Z)=7, q(Z)=0$.

Proof. Because $\chi\left(\mathcal{O}_{Z}\right)=8$ we only need to prove that $q(Z)=0$. But $q(Z)=$ $h^{1}\left(\mathcal{O}_{Z}\right)=h^{1}\left(Y, \pi_{*} \mathcal{O}_{Z}\right)=\sum_{g \in G} h^{1}\left(Y, \mathcal{O}_{Y}(g)\right)$.

Now for $g \neq 0, h^{1}\left(Y, \mathscr{O}_{Y}(g)\right)=0$. Indeed $h^{0}\left(Y, \mathscr{O}_{Y}(g)\right)=0$, because $g$ is a torsion element, $h^{2}\left(Y, \mathcal{O}_{Y}(g)=h^{0}\left(Y, \mathcal{O}_{Y}\left(K_{Y}-g\right)\right)=h^{0}\left(Y, K_{Y}+g\right)=1\right.$ by Lemma 3. Then $h^{1}\left(Y, \mathscr{O}_{Y}(g)\right)=0$ by the Riemann Roch formula.

Corollary. The elements $\xi_{1}, \xi_{2}, \xi_{3}, \eta_{1}, \eta_{2}, \eta_{3}, \zeta$ form a basis of $H^{\circ}\left(Z, K_{Z}\right)$. The canonical ring $R=\sum_{m} H^{0}\left(Z, m K_{Z}\right)$ of $Z$ is generated by these elements. There are four relations between the squares $\xi_{1}^{2}, \xi_{2}^{2}, \xi_{3}^{2}, \eta_{1}^{2}, \eta_{2}^{2}, \eta_{3}^{2}$ and $\zeta^{2}$ and any other relation in $R$ comes from these. Hence $\operatorname{Spec}(R)=\Phi_{1}(Z)=\bar{Z}$ is a complete intersection of 4 quadrics in $\mathbb{P}^{6}$ of the form $\sum_{i=1}^{3} a_{i} \xi_{i}^{2}+\sum_{i=1}^{3} b_{i} \eta_{i}^{2}+c \zeta^{2}$. Moreover $\bar{Z}$ has 24 rational double points, namely the points coming from the curves $\sigma\left(D_{j}\right)(j=1,2,3)$ on $Y$. 
Proof. Because $p_{g}(Z)=7$ and the elements $\xi_{i}(i=1,2,3)$ and $\zeta$ are independent, they form a basis of $H^{0}\left(Z, K_{Z}\right)$. Because $P_{2}(Y)=3$ and $x_{1}^{2}, x_{2}^{2}, x_{3}^{2}, y_{1}^{2}, y_{2}^{2}, y_{3}^{2}, z^{2}$ are all sections of $2 K_{Y}$ there must be 4 relations between them, hence there are 4 relations between the corresponding elements of $H^{0}\left(Z, 2 K_{Z}\right)$. Because $P_{2}(Z)=$ $\chi\left(\mathcal{O}_{Z}\right)+\frac{1}{2} \cdot 2 \cdot 1 \cdot c_{1}^{2}(Z)=8+16=24$ and there are $\left(\begin{array}{c}6+2 \\ 2\end{array}\right)=28$ quadratic monomials generated by the $\xi_{i}(i=1,2,3), \eta_{j}(j=1,2,3)$ and $\zeta$ these 4 relations are the only ones. Likewise one may prove that all the relations between higher order monomials come from these 4 ones. This proves that $\operatorname{Spec} R=\Phi_{1}(Z)$ and the assertion about the double points now follow from Mumford [9].

This corollary shows that we have proved the following theorem:

Theorem 4. The Campedelli surface $Y$ has $G=\mathbb{Z}_{2} \oplus \mathbb{Z}_{2} \oplus \mathbb{Z}_{2}$ in its torsion group. It can be realized as follows. Let $Y_{1}$ be the surface $Y$ with the three curves $D_{i}(i=1,2,3)$ blown down. The group $G$ acts on $\mathbb{P}^{6}$ (see Example 2) and $Y_{1}$ is the quotient under $G$ of an intersection of 4 quadrics in $\mathbb{P}^{6}$ each of which is $G$-invariant (i.e. a linear combination of the quadrics of the homogeneous coordinates in $\mathrm{P}^{6}$ ) and does not meet the fixed locuses of the generators of $G$. The intersection of the 4 quadrics has precisely 24 ordinary double points and no other singular points and they are distributed over three G-orbits.

Corollary. $\pi_{1}(Y) \cong \mathbb{Z}_{2} \oplus \mathbb{Z}_{2} \oplus \mathbb{Z}_{2}$, hence Tor $(Y) \cong \mathbb{Z}_{2} \oplus \mathbb{Z}_{2} \oplus \mathbb{Z}_{2}$.

Proof. We prove that $Z$ is simply connected. First of all there is a family of deformations $\left\{\bar{Z}_{t}\right\}(t \in \Delta)^{2}$ such that for $t \neq 0, \bar{Z}_{t}$ is a complete intersection of 4 quadrics in $\mathbb{P}^{6}$ without singular points and $\bar{Z}_{0} \approx \bar{Z}$. Now one applies the results of Brieskorn [4] to see that there is a family of deformations $\left\{Z_{t}\right\}(t \in \Delta)$ such that each $Z_{t}$ is a minimal resolution $\bar{Z}_{t}$, i.e. $Z_{t} \approx \bar{Z}_{t}$ for $t \neq 0$ and $Z_{0} \approx Z$. This means that $Z_{t}$ and $Z$ are the same topologically, hence $\pi_{1}(Z)=\pi_{1}\left(Z_{t}\right)=0$.

\section{§4. Deformations of Godeaux Surfaces and Campedelli's Surface}

Theorem 5. The Godeaux surfaces with torsion groups $\mathbb{Z}_{5}, \mathbb{Z}_{8}, \mathbb{Z}_{4} \oplus \mathbb{Z}_{2}$ and $\mathbb{Z}_{2} \oplus \mathbb{Z}_{2} \oplus \mathbb{Z}_{2}$ all have smooth local moduli spaces. The first one has dimension 8 , the last three have dimension 6.

Proof. Because the Godeaux surfaces are of general type $h^{0}(\Theta)=0$ for them. If we can prove that $h^{2}(\Theta)=0$ as well we find first of all that

$$
h^{1}(\Theta)=-\chi(\Theta)=10 \chi(\Theta)-2 c_{1}^{2}
$$

hence equals 8 in the first case and 6 in the other three cases. Then the statement of the local moduli spaces is a well known consequence.

We shall prove that $h^{2}(\Theta)=0$ for the last three surfaces, the proof that $h^{2}(\Theta)=0$ for the first one being similar but simpler. If $T=Q_{1} \cap Q_{2} \cap Q_{3} \cap Q_{4}$ is the complete intersection of 4 quadrics $Q_{i}(i=1,2,3)$ in $\mathbb{P}^{6}$ the statement $h^{2}\left(T, \Theta_{T}\right)=0$ will

2 As usual $\Delta$ is a small disc around the origin in $\mathbb{C}$. 
imply $h^{2}(\Theta)=0$ for the last three Godeaux surfaces. So we shall prove that $h^{2}\left(T, \Theta_{T}\right)=0$. Remark that dually one must prove that $h^{0}\left(\Omega_{T}^{1} \otimes K_{T}\right)=0$. We use the exact sequences:

$$
0 \rightarrow \stackrel{4}{\oplus} \mathscr{O}_{T}(-1) \rightarrow \Omega_{\mathbb{P} 6}^{1}(1) \mid T \rightarrow \Omega_{T}^{1} \otimes K_{T} \rightarrow 0
$$

and

$$
0 \rightarrow \Omega_{\mathbb{P} 6}^{1}(1) \mid T \rightarrow \stackrel{7}{\oplus}^{\circ} \mathcal{O}_{T} \rightarrow \bullet_{T}(1) \rightarrow 0 .
$$

The first one gives that $h^{0}\left(\Omega_{T}^{1} \otimes K_{T}\right)=h^{0}\left(\Omega_{\mathbb{P}^{6}}^{1}(1) \mid T\right)$. From the second one we have the inequality:

$$
h^{0}\left(\Omega_{\mathbb{P}^{6}}^{1}(1) \mid T\right) \leqq 7-7+h^{1}\left(\Omega_{\mathbb{P}^{6}}^{1}(1) \mid T\right) .
$$

This reduces the proof to showing that $h^{1}\left(\Omega_{\mathbb{P}^{6}}^{1}(1) \mid T\right)=0$. In fact we have a slightly more general vanishing theorem.

Lemma 5. $h^{1}\left(\Omega_{\mathbb{P}^{6}}^{\mathrm{I}}(l) \mid T\right)=0$ for $l \neq-8$.

Proof. We put $Q_{1} \cap Q_{2} \cap Q_{3}=T_{1}$ and $Q_{1} \cap Q_{2}=T_{2}$. We have exact sequences of the form:

$$
\begin{aligned}
& 0 \rightarrow \Omega_{\mathbb{P}^{6}}^{1}(k) \rightarrow \Omega_{\mathbb{P}^{6}}^{1}(k+2) \rightarrow \Omega_{\mathbb{P}^{6}}^{1}(k+2) \mid Q_{1} \rightarrow 0, \\
& 0 \rightarrow \Omega_{\mathbb{P}^{6}}^{1}(m)\left|Q_{1} \rightarrow \Omega_{\mathbb{P}^{6}}^{1}(m+2)\right| Q_{1} \rightarrow \Omega_{\mathbb{P}^{6}}^{1}(m+2) \mid T_{2} \rightarrow 0, \\
& 0 \rightarrow \Omega_{\mathbb{P}^{6}}^{1}(n)\left|T_{2} \rightarrow \Omega_{\mathbb{P}^{6}}^{1}(n+2)\right| T_{2} \rightarrow \Omega_{\mathbb{P}^{6}}^{1}(n+2) \mid T_{1} \rightarrow 0
\end{aligned}
$$

and

$$
0 \rightarrow \Omega_{\mathbb{P}^{6}}^{\mathbf{1}}(r)\left|T_{1} \rightarrow \Omega_{\mathbb{P}^{6}}^{1}(r+2)\right| T_{1} \rightarrow \Omega_{\mathbb{P}^{6}}^{1}(r+2) \mid T \rightarrow 0 .
$$

The first one and Bott's vanishing theorem (Bott, [3]) a special case of which states that $h^{1}\left(\Omega_{\mathrm{p}^{6}}^{1}(k)\right)=0$ for $k \neq 0$, gives that $h^{j}\left(\Omega_{\mathbb{P}^{6}}^{1}(k) \mid Q_{1}\right)=0$ all $k$ for $j \geqq 2$, but $h^{1}\left(\Omega_{\mathbb{P}^{6}}^{1}(k) \mid Q_{1}\right)=0$ for $k \neq-2$. Now we proceed by induction. Q.E.D.

Theorem 6. For the Campedelli surface $Y$ one has that $h^{2}\left(Y, \Theta_{Y}\right)=0$, hence $Y$ has a smooth moduli space of dimension 6 . Three dimensions come from the fact that the construction of the Campedelli surface depends on 3 parameters. The remaining three parameters come from independently letting vanish the three curves $D_{i}(i=1,2,3)$.

Proof. We closely follow the computation of the proof of Burns and Wahl, [5], Theorem (4.2). As before we let $Z_{1}$ be the intersection of 4 quadrics in $\mathbb{P}^{6}=P$ given by $F_{i}=0(i=1,2,3)$. Let $Z$ be the minimal resolution of singularities. Note that $G$ operates on $Z$ and $Z_{1}$, the respective quotients being $Y$ and $Y_{1}$. The group $G$ also operates on $H^{1}\left(\Theta_{Z}\right)$ and $H^{1}\left(\Theta_{Z_{1}}\right)$. For any $G$-vector space $H$, we denote by $H_{G}$ the $G$-invariant subspace of it. Notice that $H_{G}^{1}\left(\Theta_{Z}\right) \cong H^{1}\left(\Theta_{Y}\right)$ and $H_{G}^{1}\left(\Theta_{Z_{1}}\right) \cong$ $H^{1}\left(\Theta_{Y_{1}}\right)$.

We let $p, q, r$ be the three singular points of $Y_{1}$ and we use the same characters for three points on $Z_{1}$ in the 3 different $G$-orbits. We have the following exact 
sequences:

$$
\begin{aligned}
& 0 \rightarrow \Theta_{Z_{1}} \rightarrow \Theta_{P} \mid Z_{1} \rightarrow N_{Z_{1}}^{\prime} \rightarrow 0, \\
& 0 \rightarrow N_{Z_{1}}^{\prime} \rightarrow \stackrel{4}{\rightarrow} \mathcal{O}_{Z_{1}}(2) \rightarrow \underset{g \in G}{\oplus}\left(\mathbb{C}_{g(p)}+\mathbb{C}_{g(q)}+\mathbb{C}_{g(r)}\right) \rightarrow 0 \\
& 0 \rightarrow \mathbb{O}_{\mathbb{P}^{6}} \underset{\left(F_{1}, F_{2}, F_{3}, F_{4}\right)}{\rightarrow} \stackrel{4}{\oplus} \tilde{I}(2) \rightarrow N_{Z_{1}}^{\prime} \rightarrow 0, \\
& 0 \rightarrow \tilde{I}(2) \rightarrow \mathcal{O}_{P}(2) \rightarrow \bigoplus_{\mathbf{g} \in G}\left(\mathbb{C}_{g(p)}+\mathbb{C}_{g(q)}+\mathbb{C}_{g(r)}\right) \rightarrow 0
\end{aligned}
$$

The meaning of these sequences is precisely analogous to the corresponding sequences in the proof of Burns and Wahl, Theorem (4.2) ([5]). We let $G$ operate on the corresponding cohomology groups to get

$$
h^{1}\left(\Theta_{Y}\right)=h_{G}^{1}\left(\Theta_{Z_{1}}\right)+3=h_{G}^{0}\left(N_{Z_{1}}^{\prime}\right)-3
$$

Here we use that $H^{0}\left(N_{Z_{1}}^{\prime}\right) \rightarrow H^{1}\left(\Theta_{Z_{1}}\right)$ is surjective because all locally trivial deformations of $Z_{1}$ take place in $\mathbb{P}^{6}$ for reasons similar to the ones given in Burns and Wahl, [5].

Then

$$
\begin{aligned}
h_{G}^{0}\left(N_{Z_{1}}^{\prime}\right) & =4 h_{G}^{0}\left(\mathcal{O}_{Z_{1}}(2)\right)-3+4 h_{G}^{1}(\tilde{I}(2)) \\
& =9+4 h_{G}^{1}(\tilde{I}(2)) .
\end{aligned}
$$

Hence $h^{1}\left(\Theta_{Y}\right)=6+4 h_{G}^{1}(\tilde{I}(2))$. The last sequence shows that $h_{G}^{1}(\tilde{I}(2))=3-s$, where $s$ is the rank of the $3 \times 7$ matrix formed by the values of the seven $G$-invariant squares at the three points $p, q$ and $r$. Because $p, q$ and $r$ are in general position $s=3$ and $h^{1}\left(\Theta_{Y}\right)=6$. Then, by Riemann Roch $h^{2}\left(\Theta_{Y}\right)=0$ and $Y$ is not obstructed, in fact the local moduli space of $Y$ is smooth and of dimension 6 . The curves $D_{i}$ can be independently made to vanish and contribute each 1 parameter to the moduli space, as follows from Burns and Wahl [5]. The remaining 3 parameters then obviously come from the construction of the Campedelli surface. Q.E.D.

Corollary. The Campedelli surface can be deformed into a Godeaux surface with $\mathbb{Z}_{2} \oplus \mathbb{Z}_{2} \oplus \mathbb{Z}_{2}$ as a torsion group.

\section{References}

1. Bombieri, E.: The pluricanonical map of a complex surface. Lecture Notes in Math. 155, 35-87. Berlin-Heidelberg-New York: Springer 1970

2. Bombieri, E.: Canonical models of surfaces of general type. Publ. I.H.E.S. 42, 447-495 (1974)

3. Bott, R.: Homogeneous vector bundles. Ann. of Math. 66, 203-248 (1957)

4. Brieskorn, E.: UUber die Auflösung gewisser Singularitäten holomorpher Abbildungen. Math. Ann. 166, 76-102 (1966)

5. Burns, D., Wahl, J.: Local contributions to global deformations of surfaces. Inventiones math. 26, 67-88 (1974)

6. Campedelli, L.: Sopra alcuni piani doppi notevoli con curve di diramazione del decimo ordine. Atti Acad. Naz. Lincei 15, 358-362 (1932)

7. Godeaux, L.: Sur la construction de surfaces non rationelles de genre zero. Bull. de l'Ac. de Belge 688-693 (1949) 
8. Kodaira, K.: On the structure of compact complex analytic surfaces IV. Am. J. of Math. 90, 1048-1066 (1968)

9. Mumford, D.: The canonical ring of an algebraic surface. Ann. of Math. 76, 612 615 (1962)

10. Reid, M.: Some new surfaces with $p_{\mathrm{g}}=0$. (Preliminary version)

\section{Department of Mathematics}

Harvard University

Cambridge, Mass. 02138

USA

Present address:

Math. Instituut der R.U.

Wassenaarseweg 80

Leiden, The Netherlands

Received June 30, 1975 